\title{
Influence of Convective and Radiative Cooling on Heat Transfer for a Thin Wire with Temperature-Dependent Thermal Conductivity
}

\begin{abstract}
OKEY OSELOKA ONYEJEKWE
Robnello Unit for Continuum Mechanics and Nonlinear Dynamics, Umuagu Oshimili South, Asaba Delta State, NIGERIA

Abstract In this study, a numerical prediction of temperature profiles in a thin wire exposed to convective, radiative and temperature-dependent thermal conductivity is carried out using a finitedifference linearization approach. The procedure involves a numerical solution of a one-dimensional nonlinear unsteady heat transfer equation with specified boundary and initial conditions. The resulting system of nonlinear equations is solved with the Newton-Raphson's technique. However unlike the traditional approach involving an initial discretization in space then in time, a different numerical paradigm involving an Euler scheme temporal discretization is applied followed by a spatial discretization. Appropriate numerical technique involving partial derivatives are devised to handle a squared gradient nonlinear term which plays a key role in the formulation of the Jacobian matrix. Tests on the numerical results obtained herein confirm the validity of the formulation.
\end{abstract}

Key-words: temperature-dependent, thermal conductivity, finite difference, nonlinearity, unsteady heat transfer, Newton-Raphson technique, Jacobian matrix, Euler scheme,

Received: May 15, 2021. Revised: October 22, 2021. Accepted: December 16, 2021. Published: January 2, 2022.

\section{Introduction}

Non-linearity in heat transfer problems occurs when thermo-physical parameters are temperature -dependent or when boundary conditions are nonlinear. Examples of this occur frequently in practice for example in groundwater flow, heat exchangers, environmental pollution, fin design, biological systems etc. Although the method of separation of variables has wide applicability, it is limited to linear problems. The resulting nonlinear differential equations describing such systems are usually computed iteratively until a certain error tolerance value is satisfied. However for strongly nonlinear problems, the iterative process can diverge and cause numerical instability. Many researchers have been able to deal with this problem satisfactorily by manipulating the Jacobian matrix encountered in the Newton-Raphson (NR) method [1-7]. This approach has been facilitated by the advent of the latest generation of high speed computers.

The thin wire under consideration is assumed to be of uniform cross-section and is long enough so that temperature variation is only relevant in the axial direction. Hence heat transfer process is one-dimensional. The thermal conductivity is temperature-dependent and can be modelled by power law or linear dependency on temperature [8-11].

In this work, we shall consider the effects radiation as well as convection and nonlinear conduction in the overall heat transfer process. Radiation for example is a huge contributor to nonlinearity and its impact on the temperature is quite considerable especially in the performance of heat exchangers at high temperatures [12]. 


\section{Numerical Formulation}

Consider a heat transfer problem of a 1D conductive-convective-radiative thin metal with a fin profile area $A_{p}$, length $\mathrm{L}$, perimeter $\mathrm{P}$.

The rod has a fixed base temperature $T_{b}$ and extends into the surrounding fluid of temperature $T_{a}$. At the fin surface, heat loss occurs by convection and radiation. The energy balance for the longitudinal hotwire is given by:

$$
\begin{aligned}
& \rho c_{v} \frac{\partial T}{\partial t}=\frac{\partial}{\partial X}\left(k A \frac{\partial T}{\partial X}\right)-h P\left(T-T_{a}\right) \\
& -\varepsilon_{r} \sigma P\left(T^{4}-T_{s}^{4}\right)=0, \quad 0<X<L
\end{aligned}
$$

where $\mathrm{k}$ is thermal conductivity, $\mathrm{h}$ is the heat convection transfer coefficient, $\rho, c_{v}$ are the density and volumetric heat capacity, $\sigma$ is the Stefan-Boltzmann constant, $\mathrm{X}$ and $\mathrm{t}$ are the spatial and temporal variables, and $T$ is the temperature. It is assumed that the metal is homogeneous and isotropic, convection heat coefficient between the thin metal and the environment is constant and uniform over the entire surface of the solid. Heat dissipated from the surface obeys the Stefan-Boltzmann law. The thermal conductivity $\mathrm{k}$ is dependent on the local temperature. Other thermo-physical parameters such as the heat transfer coefficient, $\mathrm{h}$ and surface emissivity $\varepsilon_{r}$ are assumed to be constant. The boundary and initial conditions can be specified as:

$T(t, 0)=T_{b} \quad, T(t, L)=T_{L}$

Initially the metal is kept at the ambient temperature:

$T(0, X)=T_{a}$

To facilitate computation, the following dimensionless parameters are introduced:

$$
\begin{aligned}
& x=\frac{X}{L}, \quad \tau=\frac{k_{a} t}{\rho c_{v} L^{2}}, \\
& \theta=\frac{T-T_{a}}{T_{b}-T_{a}} \quad D(\theta)=\frac{k(T)}{k_{a}}, \\
& \varepsilon_{r}=\frac{\varepsilon \sigma P L^{2} T_{b}^{3}}{k_{a} A}, M^{2}=L^{2} \frac{h P}{k A}
\end{aligned}
$$

where $M^{2}$ is the thermo-geometric parameter, $k_{a}$ is the thermal conductivity of the rod at ambient temperature.

Equation (1) together with the boundary conditions are given as :

$$
\begin{aligned}
& \frac{\partial \theta}{\partial \tau}=\frac{\partial}{\partial x}\left\lfloor D(\theta) \frac{\partial \theta}{\partial x}\right\rfloor \\
& -M^{2} \theta-\varepsilon_{r} \theta^{4}, \quad 0<x<1
\end{aligned}
$$

where

$$
D(\theta)=\kappa_{0} \exp (\chi \theta)
$$

The boundary and initial conditions can also be rewritten as:

$$
\theta(0, \tau)=0, \quad \theta(1, \tau)=1, \quad \theta(x, 0)=0(4 a)
$$

and

$$
\frac{\partial^{2} \theta}{\partial x^{2}}=\frac{1}{D(\theta)}\left[-\frac{\partial D}{\partial u}\left(\frac{\partial \theta}{\partial x}\right)^{2}+M^{2} \theta+\varepsilon_{r} \theta^{4}+\frac{\partial \theta}{\partial \tau}\right]
$$

Equation (5) is a two-point nonlinear boundary value problem (TPBVP) and is solved iteratively by the NR method. For the numerical implementation we adopt the method described in [13] with some modifications. Unlike the traditional approach where the governing differential equation is first discretized in space, to yield an initial-value (Cauchy) problem involving a system of first order ordinary differential equations (ODEs); the equation is first discretized in time to obtain a sequence of TPBVPs. In order to facilitate stability, an 
implicit scheme is utilized for the temporal discretization. The finite difference method is employed for the spatial discretization. Complete discretization of the temporal and spatial components of equation (5) yields an approximate discrete equation, which is applied to each node of the problem ID domain to yield a system of algebraic equation. This allows the application a modified NR iteration technique.

Equation (5) is simply written as :

$\frac{\partial^{2} \theta}{\partial x^{2}}=f\left(\theta_{n}, v_{n}, \theta_{n-1}\right)$

where

$$
f\left(\theta_{n}, v_{n}, \theta_{n-1}\right)=\frac{\phi\left(\theta_{n}, v_{n}, \theta_{n-1}\right)}{D\left(\theta_{n}\right)}
$$

hence

$$
\begin{aligned}
& \phi\left(\theta_{n}, v_{n}, \theta_{n-1}\right)= \\
& \left(\begin{array}{l}
-\frac{\partial D(\theta)}{\partial \theta}\left(\frac{\partial \theta}{\partial x}\right)^{2}+ \\
M^{2} \theta+\varepsilon_{r} \theta^{4}+\frac{\theta_{n}-\theta_{n-1}}{\tau}
\end{array}\right)
\end{aligned}
$$

In accordance to the time discretization, the values of $\theta\left(x, \tau_{n}\right), \quad \theta\left(x, \tau_{n-1}\right)$ are given as::

$$
\begin{aligned}
& \theta_{n}=\theta_{n}(x), \\
& \theta_{n-1}=\theta_{n-1}(x)
\end{aligned}
$$

The spatial gradient is $v_{n}=\partial \theta_{n} / \partial x$

The NR iteration scheme for the computation of a nonlinear system of equation is defined as:

$$
\theta_{n}^{(k+1)}=\theta_{n}^{(k)}-\left(\mathbf{J}_{n}^{(k)}\right)^{(-1)} \mathbf{G}_{\mathbf{n}}\left(\theta_{n}^{(k)}\right)
$$

Equation (9) is a matrix equation and its manipulation can be made clearer if it is put in the form:

$$
\left(\Delta \theta_{n}^{(k+1)}\right)=-\left(\mathbf{J}_{n}^{(k)}\right)^{(-1)} \mathbf{G}_{\mathbf{n}}\left(\theta_{n}^{(k)}\right)
$$

where $\mathbf{G}_{\mathbf{n}}$ can 'loosely' be described as the right hand side (RHS) column vector of known quantities and houses the boundary and initial condition ( first guess). It is defined in [12 ] as:

$\mathbf{G}_{\mathbf{n}}=\left[G_{n, 1}, G_{n, 2} \ldots \ldots \ldots \ldots \ldots G_{n, N}\right]^{T}$

And

$G_{n, i}=$

$\theta_{n, i-1}-2 \theta_{n, i}+\theta_{n, i+1}-h^{2} f_{n, i}$

where $f_{n, i}=f\left(\theta_{n, i}, v_{n, i}, \theta_{n-1, i}\right)$

The first and last entries should reflect the boundary conditions at both ends of the problem domain

$G_{n, 1}=\theta_{n, 1}-\alpha\left(\tau_{n}\right)$,

and $G_{n, N}=\theta_{n, N}-\beta\left(\tau_{n}\right)$,

The Jacobian matrix is defined as:

$\mathbf{J}_{\mathbf{n}}^{(k)}=\frac{\partial \mathbf{G}_{\mathbf{n}}}{\partial \theta_{n}}=\left(\theta_{\mathbf{n}}^{(k)}\right)$

It is expressed at the grid points as:

$$
\begin{aligned}
& J_{n(i-1)}^{(k)}=1+1 / 2\left(h p_{n, i}^{(k)}\right), \\
& J_{n(i)}^{(k)}=-2+\left(h^{2} q_{n, i}^{(k)}\right), \\
& J_{n(i+1)}^{(k)}=1-1 / 2\left(h p_{n, i}^{(k)}\right)
\end{aligned}
$$

where

$$
\begin{aligned}
& q_{n, i}^{(k)}=q\left(\theta_{n, i}^{(k)}, v_{n, i}^{(k)}, \theta_{n-1, i}^{(k)}\right), \\
& p_{n, i}^{(k)}=p\left(\theta_{n, i}^{(k)}, v_{n, i}^{(k)}\right),
\end{aligned}
$$




$$
\begin{aligned}
q & =\partial_{\theta} f, \\
p & =\partial_{v} f, \quad \theta=\theta_{i}, \\
v & =\left(\theta_{i+1}-\theta_{i-1}\right) / 2 h
\end{aligned}
$$

The Jacobian matrix is easily computed and is found to be tridiagonal because each $\mathbf{J}_{i}(\theta)$ only depends on the grids at $i-1, i, i+1$. Hence the nonzero elements of the Jacobian matrix for rows $2,3 \ldots \mathrm{N}-1$ (the first and the last rows are apriorily determined by the boundary conditions) are computed as:

$$
\begin{aligned}
& L_{i, i-1}=\frac{\partial G_{i}}{\partial \theta_{i-1}}= \\
& 1-h^{2}\left(\frac{\partial f}{\partial \theta} \frac{\partial \theta}{\partial \theta_{i-1}}+\frac{\partial f}{\partial v} \frac{\partial v}{\partial v_{i-1}}\right) \\
& L_{i, i}=\frac{\partial G_{i}}{\partial \theta_{i}}= \\
& 1-h^{2}\left(\frac{\partial f}{\partial \theta} \frac{\partial \theta}{\partial \theta_{i}}+\frac{\partial f}{\partial v} \frac{\partial v}{\partial v_{i}}\right) \\
& L_{i, i+1}=\frac{\partial G_{i}}{\partial \theta_{i+1}}= \\
& 1-h^{2}\left(\frac{\partial f}{\partial \theta} \frac{\partial \theta}{\partial \theta_{i+1}}+\frac{\partial f}{\partial v} \frac{\partial v}{\partial v_{i+1}}\right)
\end{aligned}
$$

$L_{1,1}=L_{N, N}=1 \quad$ for Dirichlet boundary condition specifications

The various components of the partial derivatives are defined as:

$$
\begin{gathered}
q_{n}=\frac{\partial f_{n}}{\partial \theta_{n}}=\frac{1}{D\left(\theta_{n}\right)}\left(\frac{\partial \phi_{n}}{\partial \theta_{n}}-f_{n} \frac{\partial D(\theta)}{\partial \theta_{n}}\right) \\
p_{n}=\frac{\partial f_{n}}{\partial v_{n}}=\frac{1}{D(\theta)}\left(\frac{\partial \phi_{n}}{\partial v_{n}}\right)
\end{gathered}
$$

$$
\begin{aligned}
& \frac{\partial \phi_{n}}{\partial \theta_{n}}=\frac{1}{\tau}-\frac{\partial^{2} D\left(\theta_{n}\right)}{\partial x^{2}} v_{n}^{2} \\
& \frac{\partial \phi_{n}}{\partial v_{n}}=-2 \frac{\partial D(\theta)}{\partial \theta_{n}} v_{n}
\end{aligned}
$$

\section{Results and Discussion}

In the foregoing work, we have obtained physically realistic results to confirm the efficacy of our numerical technique. Equation (3) is solved for $\mathrm{N}=51$ mesh-points, $\mathrm{M}=41$ temporal grids, $\chi=0.5, \kappa_{0}=0.1, \quad$ with integration range $0<\tau<20$, for time, and $1<x<3$ for space, time step $\Delta \tau=0.25$, and spatial step $\mathrm{h}=0.05$. $M=0.5, \varepsilon_{r}=0.5$ Iteration is carried out until the difference between current and previous results satisfy a predetermined error tolerance; $\left\|\theta_{n}^{k+1}-\theta_{n}^{k}\right\|<\varepsilon$ where for this study $\varepsilon=.0001$. The results for the nonlinear diffusion heat transfer case are shown in Figs. 1a, 1b and 1c. Fig. 1a is consistent with the physics of heat transfer.

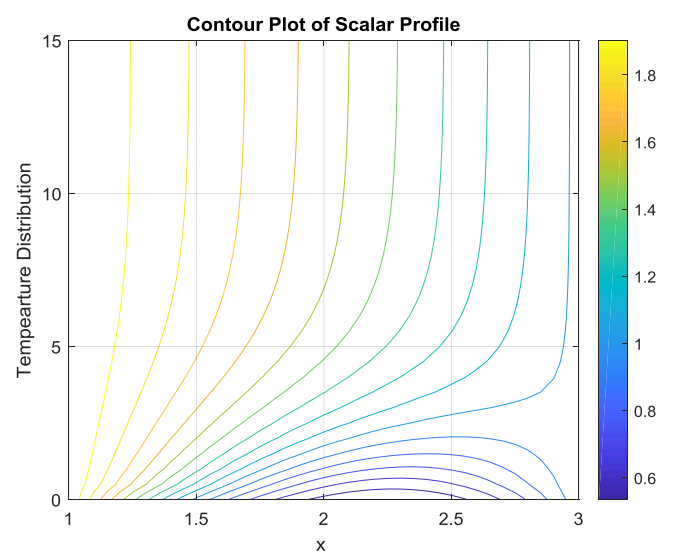

Fig. 1a : Temperature field : Nonlinear Diffusion

It can be seen that higher temperatures move from the higher to the cooler end and tend to 'smother' the effects of the lower temperature 
imposed on the right hand side (RHS) boundary. The temperature bar at the right end of the figure confirms the extent to which this happens. The domain of the cooler temperatures is confined to the right end of the rod. The 3D plot of the temperature profile shown in Fig. 1b confirms the observation in Fig. 1a. A close look reveals

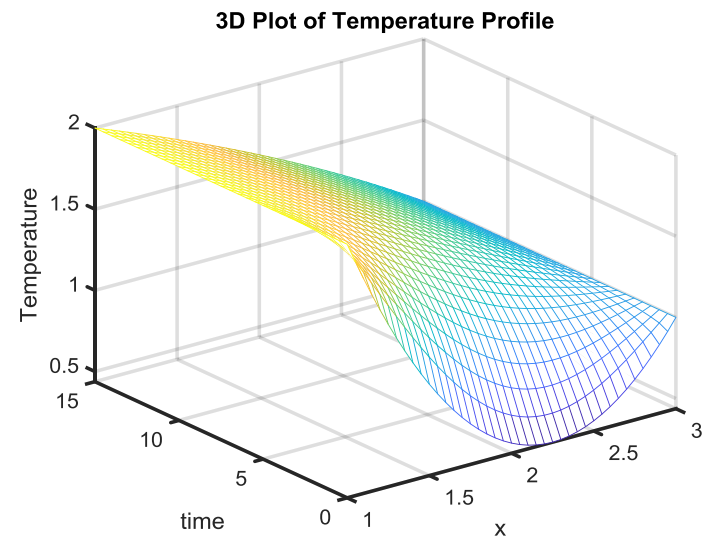

Fig.1b:3D Temperature field : Nonlinear Diffusion

that a sudden profile change (a profile separation almost) happens around $\mathrm{x}=1.5$ where the temperature profiles from the cooler end return to the $\mathrm{x}$ axis instead of progressing to the hotter end . Further progress to the hotter end would have been a contradiction to the law of conservation of energy. Fig. 1c shows that the temperature profiles become more linear as time increases.

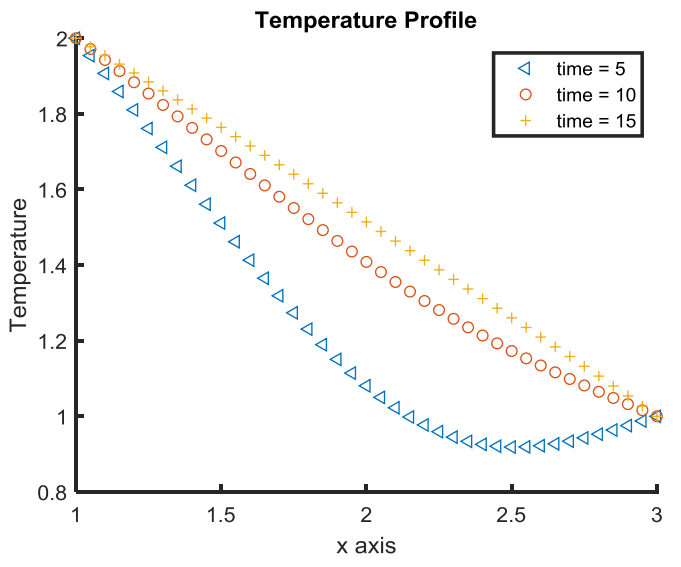

Fig. 1c: Transient Temperature field: Nonlinear Diffusion

This is not surprising because the whole system tends to steady state.

Figs. $2 \mathrm{a}, 2 \mathrm{~b}$ and $2 \mathrm{c}$ show the influence of convection and nonlinear diffusion in the heat transfer process. Starting from Fig. 2a,

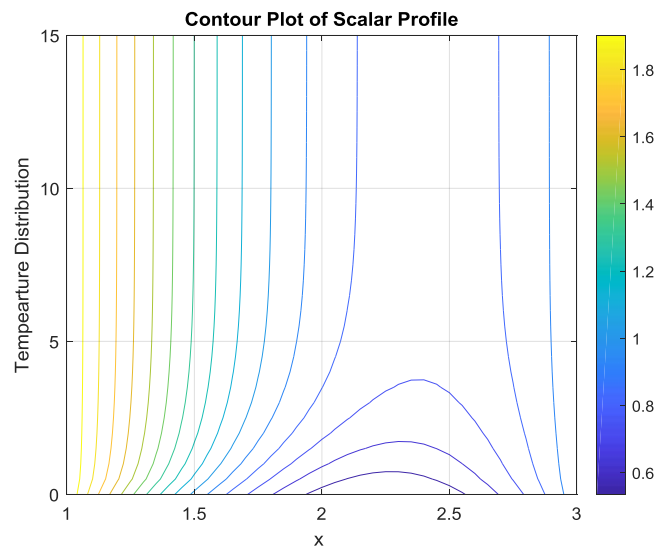

Temperature field: Nonlinear Diffusion and Convection

it is interesting to observe how the influence of the colder end on the temperature profiles seems to have changed especially in the vicinity of the RHS boundary. This is as a result of cooling by convective heat transfer. In comparison with the previous case, colder temperature profiles are observable near the right boundary. Most of this change happens within the $1.6<x<2.8$ as confirmed by Fig.2b.

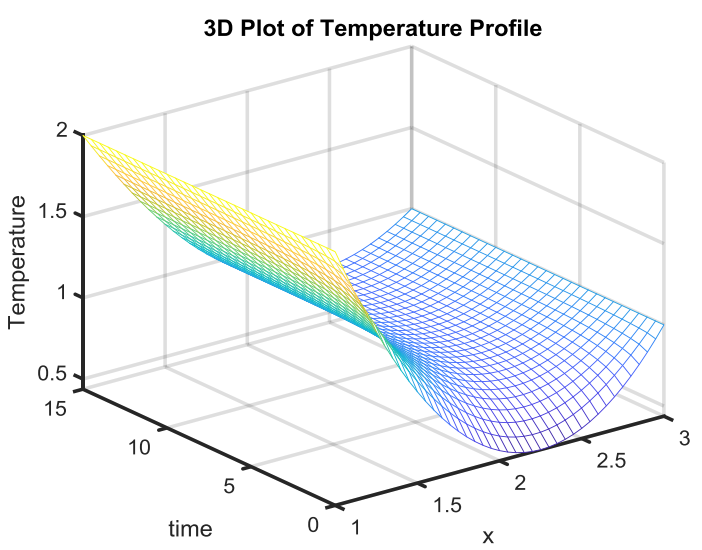


Fig. 2b : 3D Temperature profile for Nonlinear Diffusion and Convection

Fig. 2c shows that unlike Fig. 1c, there is little

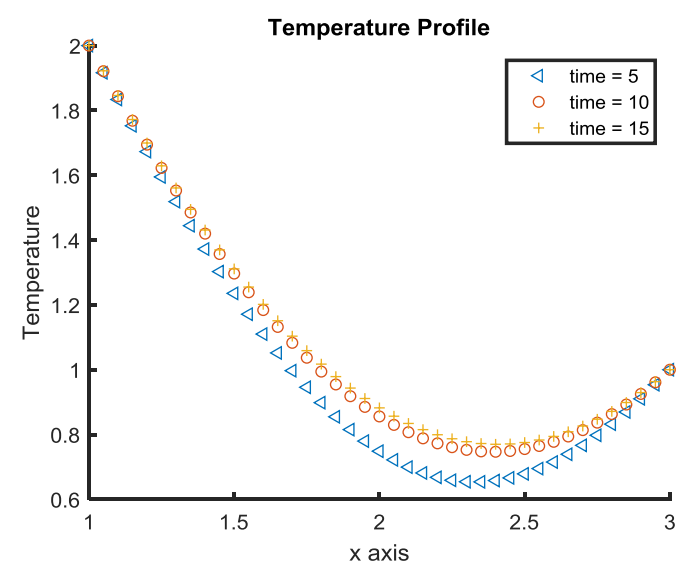

Fig.2c: Transient Temperature Profiles for Nonlinear Diffusion and Convection

change in dimensionless temperature with time especially for $\tau=5, \tau=10$. In addition, the temperatures are much less than previously as heat is convected away from the surface.

The effects of Nonlinear diffusion and radiation heat loss can be observed in Figs. 3a, 3b and 3c.

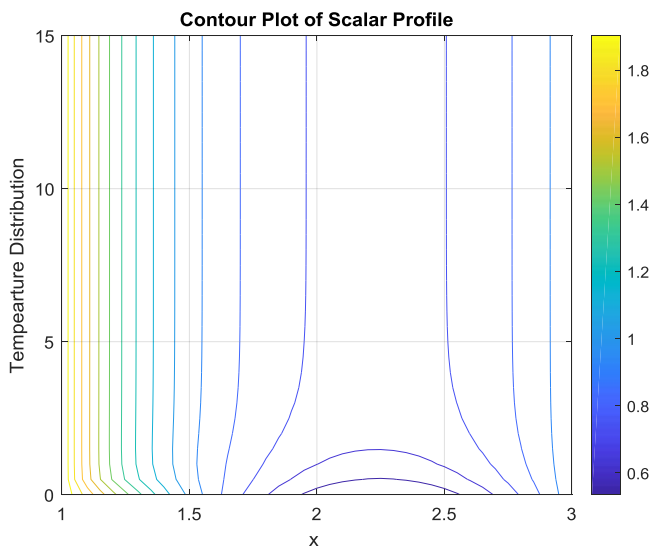

Fig. 3a Temperature field for Nonlinear Diffusion and Radiation
More heat transfer activities can now be seen to be happening at the left and right end boundaries. As the influence of the colder dimensionless temperature profiles are felt more at the RHS boundary, higher temperatures profiles are confined to the left boundary in accordance with conservation of energy. This balance is very mush shown in figure $3 \mathrm{~b}$ where

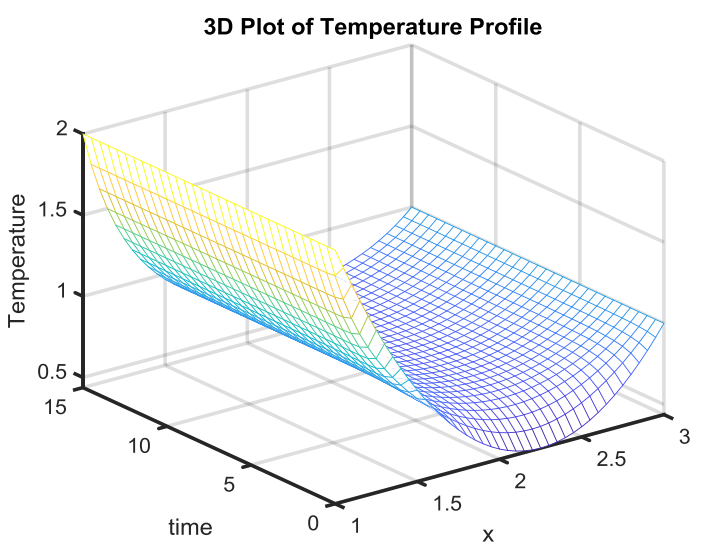

Fig. 3b: 3D Temperature Profile for Nonlinear Diffusion and Radiation

it is further demonstrated that the region of fast transitions is positioned within $1.8<x<2.7$. This is in total agreement with Fig.3a.

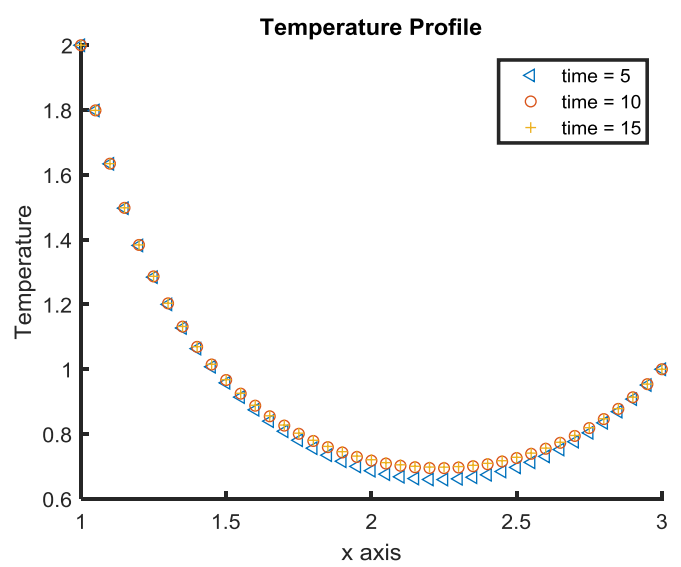


Fig. 3c: Transient Temperature field for Nonlinear Diffusion and Radiation

Fig. 3c illustrates a significant influence of radiation cooling in the overall heat transfer process. As can be observed, there is an overall cooling effect on the surface of the rod despite the time change. The cooling effect is significantly lower than in the previous cases considered. Figs. $4 \mathrm{a}, 4 \mathrm{~b}$ and $4 \mathrm{c}$ illustrate nonlinear conduction, radiation and convection effects on the overall heat transfer activity. Fig. 4a demonstrates the importance of considering both convection and radiation in the energy equation.

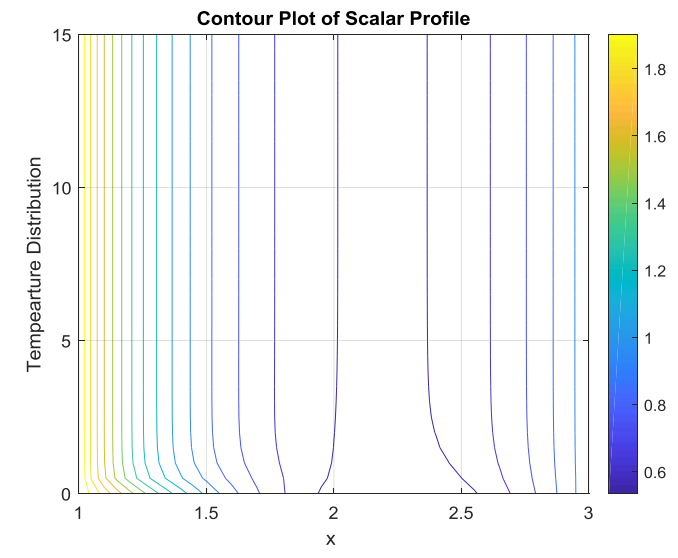

Fig. 4a: Temperature field for Nonlinear Diffusion, Convection and Radiation

The hotter temperature profiles can be seen to be moving more uniformly towards the cooler end. There are no profile singularities closer to the cooler end as was the case in previous considerations. This is confirmed in Fig. $4 \mathrm{~b}$ below.

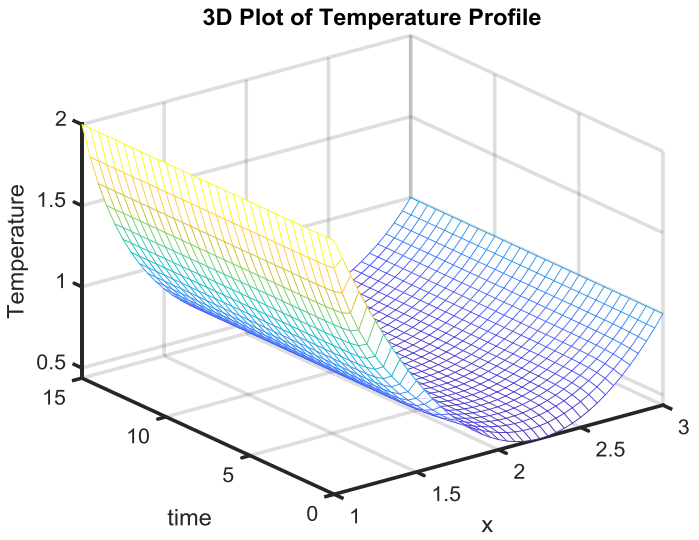

Fig. 4b: Scalar Profile for Diffusion, Convection and Radiation.

Fig. $4 \mathrm{c}$ shows that a combination of heat transfer rates resulting from nonlinear conduction, radiation and convection produce lower surface temperatures than in the previous cases.

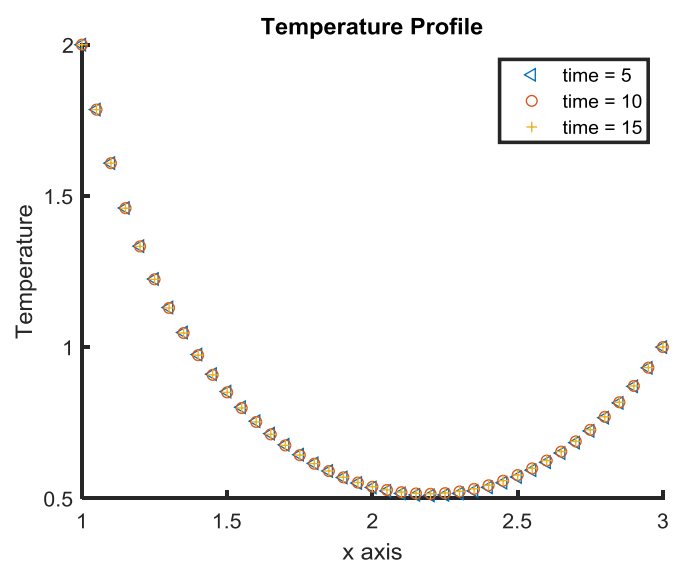

Fig.4c: Transient Temperature field for Nonlinear Diffusion, Convection and Radiation

\section{Conclusion}

In the work reported herein, heat transfer computations involving temperature-dependent thermal conductivity as well as convection and nonlinear radiation effects have been carried out using an FD modified NR approach. The results are physically plausible and amply demonstrate the impact of convection and radiation on the overall heat transfer process. Of these two (convection and radiation), radiation effects are 
more significant. It has previously been demonstrated that for temperature distribution in a metal cooled by convection and radiation, the heat loss due to radiation, contributes to $15-20$ percent of the total loss $[14,15]$. Hence it plays a significant role in improving the thermal performance of heat loss components such as fins and more importantly in devices with low heat transfer coefficients. We hasten to comment that for this particular problem the nondimensionless temperature profiles in the region $1.3<x<2.7$ needs further study in terms of relating it to the influence of radiation parameter $\varepsilon_{r}$ thermogeometric parameter $\mathrm{M}$, conduction parameters $\kappa_{0}, \chi$. A look at the profiles within this region, suggests that certain combinations of these parameters may lead to physically unrealistic results and numerical instability which may significantly affect design.

\section{References}

[1] U.M. Ascher, R.M.M. Mattjeij, R.D. Russel, Numerical solution of boundary value problem for ordinary differential equations in; Claasics in Applied Mathematics, Vol. 13, 1995, SIAM, Philadelphia

[2] A. Marasco, A. Romano, Scientific computing with Mathematica: mathematical problems for ordinary differential equations in: Modelling and Simulation in Science, Engineering and Technology,2001, Birkhauser Boston, MA

[3] R.E. White, Computational mathematics: models, methods, and analysis with MATLAB and MPI, 2004, Chapman and Hall/CRC, Boca Raton, London, NY Washington.

[4] A.U. Keskin, Boundary value problems for engineers with MATLAB solutions, Springer Nature Switzerland, ISBN 978-3-030-21079-3 2019

[5] I. Farago Numerical methods for ordinary differential equations, TypoTech, Budapest, http://faragois.web.elte.hu/ODE_angol.pdf 2014
[6] J. Lambers, Finite difference methods for two=point boundary value problems. MAT 461/561, lecture 26 notes, department of mathematics, university of southern Mississippi http://www.math.usm.edu/lambersmat $461 /$ spr10 lecture26.pdf 2010

[7] O.O. Onyejekwe, Localized boundarydomain integro-partial differential formulations for transient scalar transport problems, International journal of applied and computational mathematics Vol. 2,No.3, 2016 DOI 10.1007/s40819-016-0235-y

[8] G. Grarslan, Numerical modelling of linear and nonlinear differential equations y compact finite difference method, Applied Mathematics and Computation, Vol. 216, 2010 pp. 2472-2478

[9] R.J.Moitsheki, T. Hayat, M. Y. Malik, Some exact solution of the fin problem with power temperature-dependent thermal conductivity, Nonlinear Analysis, Real World Appl. Vol.11, No.5 2010, pp. 3287-3294

[10] J. Hristov, Integral solutions to transient nonlinear heat (mass) diffusion with power law diffusivity: a semi-infinite medium with fixed boundary conditions, Heat Mass Transfer Vol. 52 No.3 2016, pp. 635-655

[11] A.S.Vatsala, T.G. Melton, Generalized quasilinearization method for higher order of convergence for second-order boundary-value problems, Bound. Value. Problems, 2006, pp. 115

[12] Y. Huang, X-F. Liang Exact and approximate solution of convective -radiative fins with temperature dependent thermal conductivity using integral equation method, International Journal of Heat and Mass Transfer, Vol. 150, 2020, pp. 1-8

[13] S.M. Filipov, I.D. Gospodinov and I. Farago, Replacing the finite difference methods for nonlinear two-point boundary value problems by successive application of the linear shooting method, Journal of Computational and Applied Mathematics, Vol. 358, 2019, pp.46-60 
[14] A.S. Dogonchi, M. Waqas S.M. Seyyedi, M. Hashemi-Tilehnoee, D.D. Ganji, CVFEM analysis for $\mathrm{Fe}_{3} \mathrm{O}_{4}-\mathrm{H}_{2} \mathrm{O}$ nanofluid in an annulus subject to thermal radiation, Int. Jnl. Heat Mass Transf Vol 132, 2019, pp. 473-483

[15] A.S. Dogonchi, S. Hashim, Heat transfer by natural convection of $\mathrm{Fe}_{3} \mathrm{O}_{4}-\mathrm{H}_{2} \mathrm{O}$ nanofluid in annulus between a wavy-circular circular cylinder and a rhombus, Int. Jnl. Heat Mass Transf. Vol. 130, 2019, pp. 320-332
Creative Commons Attribution License 4.0 (Attribution 4.0 International, CC BY 4.0)

This article is published under the terms of the Creative Commons Attribution License 4.0

https://creativecommons.org/licenses/by/4.0/deed.en_US 